\title{
Clearance (née Rowland) concepts: a downdate and an update
}

\author{
Leslie Z. Benet
}

Received: 2 February 2010/ Accepted: 13 November 2010/Published online: 27 November 2010

(C) The Author(s) 2010. This article is published with open access at Springerlink.com

\begin{abstract}
A number of experimental observations in the late 1960s, early 1970s could not be explained by the pharmacokinetic theory available at that time. For example rats receiving phenobarbital as an enzyme inducing agent exhibited increased elimination of phenylbutazone in vitro in liver microsomes and in vivo in whole animals compared to that observed in non-induced animals. However, for desipramine, although phenobarbital increased elimination in microsomes, no change in plasma disappearance was noted in vivo for this drug between rats induced with phenobarbital and control rats. Similar in vitro-in vivo discordancies were seen with changes in protein binding. The introduction of clearance concepts in the early 1970s by Professor Rowland and others provided the scientific rationale for these apparently contradictory findings and the recognition that clearance, not half-life, was the measure of the body's ability to eliminate drugs and most importantly that changes in pathology and physiology could be correlated with measures of clearance. Up to that time half-life was well recognized in terms of basic chemical principles as an appropriate measure of the rate of elimination and reflective of changes in the rate of elimination. The difference between chemistry and pharmacokinetics, however, is that in chemistry the volume in which the reaction occurs does not change. In contrast, in pharmacokinetics, disease states and differences in physiology can change the space available in which the drug may distribute in the body. Thus, it was necessary to develop a pharmacokinetic measure of volume that was independent of elimination, i.e., $\mathrm{V}_{\mathrm{ss}}$. Now, the relationship between $\mathrm{V}_{\mathrm{ss}}$ and clearance led to a unique measure of time of drug in the body, the mean residence time. Although this parameter is calculated in all PK programs, very few pharmaceutical scientists know how it can be useful. Very recently, we have shown
\end{abstract}

L. Z. Benet $(\bowtie)$

Department of Bioengineering and Therapeutic Sciences, Schools of Pharmacy and Medicine,

University of California San Francisco, 533 Parnassus Avenue, Room U-68, San Francisco,

CA 94103-0912, USA

e-mail: leslie.benet@ucsf.edu 
that the concepts of accumulation, prediction of which is the clinically relevant use for half-life and mean residence time, are flawed and that the appropriate time dependent parameter to predict accumulation has not been previously correctly identified. Finally, when clearance concepts were developed our understanding of the importance of drug transporters was nonexistent. A critical, and generally unrecognized assumption (which is only explicitly stated in Professor Rowland's seminal 1973 paper), in the development of the theory of clearance is that the unbound drug concentration in the organ of elimination is in a constant equilibrium with the unbound drug concentration in the systemic circulation, where drug concentration measurements are made. Transporter drug-drug and disease interactions may, in fact, change this equilibrium and potentially what we consider as intrinsic clearance, may not be independent of an eliminating organ volume parameter, contrary to what we have been teaching for the past 37 years.

Keywords Clearance - Volume of distribution - Mean residence time Multiple dosing half lives

\section{Introduction}

I am pleased and honored to participate in this dedicatory issue honoring Professor Malcolm Rowland's 70th birthday and his outstanding contributions to the fields of pharmacokinetics and pharmacodynamics. Reviewing the pharmacokinetic literature 40 years ago almost all published studies were carried out with salicylic acid. This was because the drug was given in large doses, and primarily because we had a colorimetric assay, using the Trinder method, that allowed us to measure plasma and urinary concentrations. Professor Rowland had 11 such publications between 1966 and 1972 investigating the pharmacokinetics of salicylic acid. However, the mathematical models that were developed and the equations that accompanied these models, although of interest to the cognoscente, were incomprehensible to clinicians treating patients. Furthermore, there appeared to be no useful relationship between the changes in these pharmacokinetic parameters and the degree of disease, which would allow translation of pharmacokinetics into patient drug dosing.

\section{Clearance}

So, in 1972, what was wrong with pharmacokinetics? It appeared to have no relationship with clinically meaningful parameters that could help in making drug dosing decisions or that could account for differences in physiology and pathology. For example at steady state:

$$
\text { Rate In = Rate Out }
$$

Availability $\times$ Dosing Rate $=? ? \times$ Average Concentration

$\mathrm{F} \times$ Dosing Rate $=? ? \times$ Target Concentration 
It was well known that at steady state the Rate In would be the dosing rate at which the drug was administered multiplied by the bioavailability $(\mathrm{F})$, which could change as a function of the route of administration. It was recognized that Rate Out should relate to systemic concentrations or to a target concentration that was known to yield efficacy with minimum toxicity. However, the parameter that was to be multiplied by this systemic target concentration was undefined in 1972. Therefore, Professor Rowland and others applied a term from physiology to whole body drug elimination and called it clearance (CL). So that at steady state:

$$
\text { Rate In = Rate Out }
$$

Availability $\times$ Dosing Rate $=$ Clearance $\times$ Concentration at steady - state

$$
\mathrm{F} \times \operatorname{Dose} / \tau=\mathrm{CL} \times \mathrm{C}_{\mathrm{ss}}
$$

A number of experimental observations in the late 1960s, early 1970s could not be explained by the pharmacokinetic theory available at that time. For example von Bahr et al. [1] observed that for rats receiving phenobarbital as an enzyme inducing agent the elimination of phenylbutazone was increased both in vitro in liver microsomes and in vivo in whole animals versus that observed in non-induced animals. However, for the drug desipramine, although elimination was increased in microsomes from phenobarbital induced rats, no change in plasma disappearance was noted in vivo for this intravenous dosed drug between rats induced with phenobarbital and control rats. That is, in some cases elimination of the drug was increased in both the microsomes and in vivo, but in other cases in vitro induction was seen, but no in vivo induction. Another series of studies related to protein binding also showed discordancies for certain drugs between in vitro and in vivo studies. Krüger-Thiemer et al. [2] had investigated the renal elimination of highly protein bound sulfanilamides in healthy volunteers. They showed in vitro that inhibition of protein binding would increase free concentrations of these drugs. They reasoned, therefore, that in vivo they would expect the renal elimination of these sulfanilamides to increase when protein binding was inhibited. For some of the sulfanilamides this in vivo increase in renal elimination was observed, however, for a number of sulfanilamides no change in renal elimination was found when free concentrations of the drugs were increased by inhibiting protein binding. Thus, it appeared in the early 1970s that pharmacokinetics did not provide any predictability of changes in elimination based on induction of metabolic enzymes or through increasing free drug concentrations.

However, the introduction of clearance concepts in pharmacokinetics by Rowland et al. [3] and the further explication by Wilkinson and Shand [4] alleviated these problems. Rate of elimination for an individual organ can be defined as the product of blood flow to that organ $(\mathrm{Q})$ and the difference between the arterial $\left(\mathrm{C}_{\mathrm{A}}\right)$ and venous $\left(\mathrm{C}_{\mathrm{V}}\right)$ concentrations as shown below:

$$
\text { Rate of elimination }=\mathrm{Q} \cdot \mathrm{C}_{\mathrm{A}}-\mathrm{Q} \cdot \mathrm{C}_{\mathrm{V}}
$$

From Eq. 1, organ clearance equals the rate of elimination divided by the concentration as shown in Eq. 2, where the difference in arterial and venous concentrations divided by the incoming arterial concentration may be defined as the extraction ratio (ER) of the organ. 


$$
\begin{aligned}
\mathrm{CL}_{\text {organ }} & =\left(\mathrm{Q} \cdot \mathrm{C}_{\mathrm{A}}-\mathrm{Q} \cdot \mathrm{C}_{\mathrm{V}}\right) / \mathrm{C}_{\mathrm{A}} \\
& =\mathrm{Q} \cdot\left(\mathrm{C}_{\mathrm{A}}-\mathrm{C}_{\mathrm{V}}\right) / \mathrm{C}_{\mathrm{A}}=\mathrm{Q} \cdot \mathrm{ER}
\end{aligned}
$$

However, Eq. 2 would not explain the anomalies listed above with respect to enzyme induction and protein binding. Thus the development of clearance in pharmacokinetics $[3,4]$ was advanced by describing the ER in terms of the "wellstirred" model that we borrowed from the chemical engineers as used by them in modeling the "cracking" of petroleum to make gasoline. We define ER as a function of three parameters:

a. blood flow to the elimination organ

b. the intrinsic ability of the organ to eliminate the unbound drug as if there were no flow and protein binding limitations

c. the fraction of the drug unbound in the blood.

Rowland et al. [3] introduced parameters $a$ and $b$ in their definition of the organ clearance of unbound drug. Wilkinson and Shand [4] added the protein binding parameter to allow conversion for total drug clearance characterization. In terms of the well stirred model, clearance (with respect to blood concentrations) for the eliminating organ then becomes:

$$
\mathrm{CL}_{\text {organ }}=\mathrm{Q} \cdot\left(\mathrm{fu}_{\mathrm{b}} \cdot \mathrm{CL}_{\mathrm{int}}\right) /\left(\mathrm{Q}+\mathrm{fu}_{\mathrm{b}} \cdot \mathrm{CL}_{\mathrm{int}}\right)
$$

where $\mathrm{CL}_{\mathrm{int}}$ is the intrinsic unbound clearance by the organ and $\mathrm{fu}_{\mathrm{b}}$ is the unbound fraction of drug in blood. Equation 3 demonstrates that when the capability of the eliminating organ to metabolize the drug is large in comparison to the rate of drug presentation to the organ, i.e., $\left(\mathrm{fu}_{\mathrm{b}} \cdot \mathrm{CL}_{\mathrm{int}}\right) \gg \mathrm{Q}$, the clearance will approximate the organ blood flow

$$
\mathrm{CL}_{\text {organ }} \cong \mathrm{Q}
$$

That is, drug elimination is limited by blood flow rate and the compound is called a high-ER drug. On the other hand, when the metabolic capacity is small in comparison to the rate of drug presentation, i.e., $\mathrm{Q} \gg\left(\mathrm{fu}_{\mathrm{b}} \cdot \mathrm{CL}_{\mathrm{int}}\right)$, the clearance will be proportional to the unbound fraction of drug in blood and the intrinsic clearance, as in Eq. 5.

$$
\mathrm{CL}_{\text {organ }} \cong \mathrm{fu}_{\mathrm{b}} \cdot \mathrm{CL}_{\mathrm{int}}
$$

The drug is then called a low-ER drug. When the capability for elimination is of the same order of magnitude as the blood flow, clearance is dependent upon the blood flow as well as on the intrinsic clearance and the blood protein binding (Eq. 3).

Note that the definitions for low- and high-ER drugs are independent of the fraction of the dose eliminated by a particular organ. For example, diazepam is eliminated almost completely by hepatic metabolism (less than $1 \%$ of the drug is excreted unchanged in the urine), yet the clearance of diazepam, $27 \mathrm{ml} / \mathrm{min}$, indicates that this is a low hepatic ER drug. That is, on each pass through the liver only a small fraction of the drug $\left(\mathrm{ER}_{\mathrm{H}}=\mathrm{CL}_{\mathrm{H}} / \mathrm{Q}_{\mathrm{H}}=27 / 1,500=0.018\right)$ will be eliminated, although eventually almost all of the drug will be eliminated by the liver. The value of 1,500 is the average hepatic blood flow $\left(\mathrm{Q}_{\mathrm{H}}\right)$ in $\mathrm{ml} / \mathrm{min}$ for a $70 \mathrm{~kg}$ man in calculating the hepatic $\mathrm{ER}\left(\mathrm{ER}_{\mathrm{H}}\right)$. 
Equation 3 clarifies a number of the unresolvable experimental results described above. For example, following intravenous dosing, enzyme induction or hepatic disease may change the rate of desipramine metabolism in a hepatic microsomal enzyme system, but no change in clearance is found in the whole animal with similar hepatic changes. This is explained by the fact that desipramine is a highextraction-ratio drug and clearance becomes limited by blood flow rate (Eq. 4), so that changes in $\mathrm{CL}_{\mathrm{int}}$ due to enzyme induction or liver disease have little effect on clearance. Also, although desipramine is a relatively highly protein bound drug $\left(\mathrm{fu}_{\mathrm{b}}=0.18\right)$, changes in protein binding due to disease or competitive binding should have little effect on clearance. In contrast, for a low-extraction-ratio drug such as phenylbutazone $(\mathrm{CL}=1.6 \mathrm{ml} / \mathrm{min} / 70 \mathrm{~kg})$, enzyme induction or changes in protein binding $\left(\mathrm{fu}_{\mathrm{b}}=0.039\right)$ should markedly affect elimination since Eq. 5 describes this drug's elimination following intravenous dosing.

A second extremely insightful and influential paper published by Professor Rowland in Journal of Pharmaceutical Sciences in 1972 was entitled, "The Influence of Route of Administration on Drug Availability" [5]. This paper contains the first physiologic based quantitative prediction of first-pass hepatic elimination to appear in the pharmacokinetic literature.

$$
\mathrm{F}_{\mathrm{H}}=1-\mathrm{ER}_{\mathrm{H}}=1-\mathrm{CL}_{\mathrm{H}} / \mathrm{Q}_{\mathrm{H}}
$$

I utilized this equation earlier to calculate the ER of diazepam, but it is more instructive to utilize Eq. 6 in terms of labetalol. Labetalol is a high-ER drug with a hepatic blood clearance of $1,290 \mathrm{ml} / \mathrm{min}$ in a $70 \mathrm{~kg}$ human and a drug with an oral bioavailability of only $18 \%$. Inserting the labetalol hepatic blood clearance and the average hepatic blood flow into Eq. 6 gives an $\mathrm{ER}_{\mathrm{H}}=0.86$ Thus, from Eq. 6 the hepatic bioavailability of labetalol is only $14 \%$ with the great majority of the oral dose being metabolized on its first pass through the liver prior to reaching the systemic circulation. Thus, it can be seen that the low oral bioavailability of labetalol is only due to physiology, i.e., giving the drug orally whereby it must first pass through the liver before reaching the systemic circulation (note: the small differences between the predicted $\mathrm{F}_{\mathrm{H}}$ and the actual oral bioavailability are due to the fact that the numbers here are for average subjects and coefficients of variation for labetalol clearance and bioavailability are 40 and 30\%, respectively). Equation 6 provides very useful information concerning labetalol that although the drug has poor oral bioavailability, it is not due to dosage form defects, permeability problems or intestinal metabolism. Rather giving labetalol by the oral route of administration leads to a significant decrease in hepatic first-pass bioavailability that can only be overcome by choosing an alternate route of administration.

The introduction [3] of clearance concepts to pharmacokinetics beginning in 1973 has had an immense effect on the field. Reviewing PubMed for the term "drug clearance" one finds in 1972 that there were 192 references, many of them dealing with mucociliary drug clearance. In the year 2006 the total number of references increased to more than 29,000 and as of the time of the oral presentation of this work (October 5, 2009) the number of references was 47,827. At submission of the final version for publication (November 4, 2010) the number of references has increased to 50,199. Thus, beginning in 1973 it was recognized that clearance, not 
half-life, was a measure of the body's ability to eliminate drug and changes in pathology or physiology could be correlated with measures of clearance.

\section{Volume of distribution}

This initially created some confusion because up to that time half life was well recognized in terms of basic chemical principles as an appropriate measure of the rate of elimination and reflective of changes in the rate of elimination. However, the difference between chemistry and pharmacokinetics is that in chemistry the volume in which the reaction occurs does not change. In contrast, in pharmacokinetics, disease states and differences in physiology can change the space available in which the drug may distribute in the body. Thus, it was necessary to develop a measure of volume of distribution that was independent of elimination. Such a volume term has been defined as volume of distribution at steady state $\left(\mathrm{V}_{\mathrm{ss}}\right)$. Although clearance could be determined independent of the previously employed pharmacokinetic models by determining the dose divided by the area under the curve (AUC), no noncompartmental method for determining $\mathrm{V}_{\mathrm{ss}}$ was available until 1979. Then Benet and Galeazzi [6] defined a noncompartmental method for determination of $\mathrm{V}_{\mathrm{ss}}$. This paper was the first to describe the relationship between $\mathrm{V}_{\mathrm{ss}}, \mathrm{CL}$ and a measure of time of drug in the body, the mean residence time (MRT).

$$
\mathrm{V}_{\mathrm{ss}}=\mathrm{CL} \cdot \mathrm{MRT}
$$

Now it was recognized that clearance and volume were the independent parameters and that half life or MRT was the dependent parameter. Changes in either clearance or volume could change half life. The work of Klotz et al. [7] studying the effects of age on the disposition of diazepam nicely demonstrates this point. Terminal plasma half life of diazepam exhibited a striking age dependence going from about $20 \mathrm{~h}$ for 20 year old men and increasing linearly with age to about $90 \mathrm{~h}$ at 80 years. The plasma clearance of diazepam showed no significant age dependence. The change in half life is due to the redistribution of body fluids with age causing more diazepam to distribute out of the fluids delivered to the liver, the organ of elimination. Since less drug is available to the liver, drug remains in the body longer and half life increases, but the elimination process has not been affected. Another excellent example of clearance and volume not changing in parallel, so that half life changes don't reflect clearance changes is the data for digoxin. Everyone today uses the equations of Sheiner et al. [8] who suggested how to predict $\mathrm{CL}$ and $\mathrm{V}$ in patients with varying renal function, either exhibiting or not exhibiting marked congestive heart failure.

However, there are relatively few well documented cases in the literature of the clearance and volume discordance, probably because no clinician would make dose adjustments (mg/day) based on volume of distribution changes. Dose adjustments are made based on clearance changes as given in Eq. 1. Therefore, very few investigators calculate the volume changes and when they do, they seldom focus even on significant changes because they are immaterial in making dose adjustments. There is now, however, beginning to be a significant literature base where this information is 
available and that relates to drug-drug interactions that are transporter based. This is because changes in transporter activity would be expected to change drug distribution characteristic as well as metabolism changes (the latter due to transporter-enzyme interplay [9]). This is in contrast to enzymatic drug-drug interactions where no change in volume would be expected. Human studies of inhibition of hepatic OATP transporter uptake for atorvastatin [10] and particularly glyburide [11] show that significant changes in volume do not parallel changes in clearance and thus half life changes would not be useful in predicting dose adjustments. Recognizing that volume would change in transporter interaction studies, Grover and Benet [12] summarized the effects of drug transporters on volume of distribution. All of these interaction studies provide a database to confirm the relationship depicted in Eq. 7 and the lack of parallel changes of clearance and half life.

Thus in summary from Eq. 7, clearance is a measure of the body's ability to eliminate drug. Volume of distribution is a measure of the space available in the body in which a drug may distribute. Pathology and physiology can change both CL and $\mathrm{V}_{\mathrm{ss}}$ thereby changing MRT or half life. So the question then becomes why do we want to know half life?

\section{Half life}

I believe that the only clinically relevant use of half life is to predict accumulation upon multiple dosing. That is, knowing the therapeutically beneficial drug dosing rate, based on clearance and bioavailability (Eq. 1), what is the appropriate dosing interval to maximize efficacy and minimize toxicity? What half life do we use to make that calculation? I have come to realize that none of the accumulation equations, which we now use, correctly predict drug accumulation. Recently, Sahin and Benet [13] defined a new parameter the "Operational multiple dosing half life" as equal to the dosing interval at steady state where the maximum concentration at steady state is twice the maximum concentration found for the first dose and where the fall off of plasma concentrations during a dosing interval at steady state is reflective of this half life. We demonstrated for diazepam that the well accepted concept that the terminal half life representing the great majority of the AUC will govern accumulation, can be incorrect. Using oral diazepam, we demonstrated that the operational multiple dosing half life is remarkably sensitive to the absorption half life even when this absorption half life is much less than the terminal half life and describe the relevance of this in designing extended release dosage forms [13].

Consider now telbivudine $\left(\right.$ Tyzeka $^{\circledR}$ ) the Novartis drug for chronic hepatitis B. Telbivudine has a distribution phase half-life of $2.0 \mathrm{~h}$ and a terminal elimination half-life of $54 \mathrm{~h}$ [14]. Yet, at steady-state with once daily dosing, peak to trough ratios are more than 10-fold. The package insert reads: Steady-state was achieved after approx. 5-7 days of once daily oral dosing with $\sim 1.5$-fold accumulation, suggesting an effective half-life of $\sim 15 \mathrm{~h}$ [14]. From Eq. 7 the half-life for the MRT in the body is $16.3 \mathrm{~h}$ and I calculate that the absorption half-life of telbivudine is approximately $1.4 \mathrm{~h}$. Using these parameters I calculated the steady state dosing intervals that would yield two-fold increases for the maximum concentration at 
Table 1 Telbivudine dosing intervals yielding ratios of 2.0 for the respective parameters as a function of absorption halflife

\begin{tabular}{lll}
\hline Parameter & \multicolumn{2}{l}{ Dosing interval $(\mathrm{h})$} \\
\cline { 2 - 3 } & $\mathrm{t}_{1 / 2, \mathrm{ka}}=1.4 \mathrm{~h}$ & $\mathrm{t}_{1 / 2, \mathrm{ka}}=7.0 \mathrm{~h}$ \\
\hline $\mathrm{C}_{\mathrm{max}, \mathrm{ss}} / \mathrm{C}_{\mathrm{max}, \mathrm{sd}}=2.0$ & 4.9 & 12.4 \\
$\mathrm{AUC}_{\mathrm{ss}} / \mathrm{AUC}_{\mathrm{sd}}=2.0$ & 5.4 & 13.6 \\
$\mathrm{C}_{\mathrm{max}, \mathrm{ss}} / \mathrm{C}_{\mathrm{min}, \mathrm{ss}}=2.0$ & 7.1 & 12.0 \\
\hline
\end{tabular}

steady state $\left(\mathrm{C}_{\max , \mathrm{ss}}\right)$ relative to the maximum concentration from a single dose $\left(\mathrm{C}_{\text {max,sd }}\right)$, the area under the curve during a dosing interval at steady state $\left(\mathrm{AUC}_{\mathrm{ss}}\right)$ to the area under the curve over the same time interval for a single dose $\left(\mathrm{AUC}_{\mathrm{sd}}\right)$ and at steady state, the ratio of the maximum concentration $\left(\mathrm{C}_{\text {max,ss }}\right)$ to the minimum concentration $\left(\mathrm{C}_{\mathrm{min}, \mathrm{ss}}\right)$, as given in Table 1. I calculate the ratio for a two-fold increase because this yields a measure of the accumulation half-life for each of the parameters. That is, if telbivudine was dosed every $7.1 \mathrm{~h}$ at steady state the maximum to minimum concentration ratio would be 2.0 and if it was dosed every $14.2 \mathrm{~h}$ the ratio would approximate 4 . Since the drug is given once daily, one can understand now why peak to trough ratios at steady state are more than 10 fold since we are dosing at intervals midway between 3 and 4 dosing interval half lives for this parameter. Note in Table 1 that if the absorption half-life could be slowed to $7 \mathrm{~h}$ then the peak to trough ratios would be approximately 4 at steady state for once daily dosing, since the accumulation half life for this parameter is now $12.0 \mathrm{~h}$.

\section{Clearance again}

Let's return to clearance. With the recognition of the importance of drug transporters during the last decade, how do we modify the clearance equations to account for uptake and efflux transporters and particularly for transporter-enzyme interplay? One generally unrecognized component of the clearance equations developed by Professor Rowland is the assumption, that is really only explicitly stated in the 1973 paper [3], that the partition ratio of free drug between the circulating systemic fluids and the fluids within an eliminating organ is a constant (usually equal to 1). Is this true when transporters are inhibited (or knocked out) or induced?

Although knock-out animals have given us great insight into understanding the importance of a variety of enzymatic and transporter disposition processes, they cannot be trusted to provide quantitative pharmacokinetic characterization of these processes. This is particularly true for evaluating enzyme-transporter interplay. In general, investigators assume that knocking out one process does not affect another in terms of quantitative clearance measurements. However, if this is not explicitly tested, the assumptions are highly questionable, especially for transporter-enzyme interactions. In essence, $\mathrm{CL}_{\text {other }}$ must be shown to be constant. 
Recently, Kusihara and Sugiyama [15] have developed a methodology to evaluate hepatic clearance measures with the addition of transporters. They define:

$$
\mathrm{CL}_{\text {int }}=\mathrm{CL}_{\text {int,met }}+\mathrm{PS}_{\text {int,bile }}
$$

where $\mathrm{PS}_{\text {int,bile }}$ is the intrinsic efflux clearance of unbound drug into the bile, while $\mathrm{CL}_{\text {int,met }}$ is the intrinsic metabolic clearance of unbound drug in the liver.

Now the intrinsic organ clearance $\left(\mathrm{CL}_{\mathrm{int}, \mathrm{org}}\right)$ is:

$$
\mathrm{CL}_{\text {int,org }}=\left[\mathrm{PS}_{\mathrm{inf}} /\left(\mathrm{PS}_{\mathrm{eff}}+\mathrm{CL}_{\mathrm{int}}\right)\right] \cdot \mathrm{CL}_{\mathrm{int}}
$$

where $\mathrm{PS}_{\mathrm{inf}}$ and $\mathrm{PS}_{\mathrm{eff}}$ represent the intrinsic clearances for cellular uptake (influx) and efflux into the systemic circulation, respectively.

At the boundary condition where $\mathrm{CL}_{\mathrm{int}} \gg \mathrm{PS}_{\text {eff }}$, i.e., where the great majority of drug taken up by the hepatocytes is metabolized or excreted into bile, then $\mathrm{CL}_{\text {int,org }}$ in Eq. 9 can be approximated by :

$$
\mathrm{CL}_{\text {int,org }} \cong \mathrm{PS}_{\text {inf }}
$$

Equation 10 appears to be consistent with the data for our atorvastatin isolated perfused rat liver study [16], our in vivo rat studies [17] and our human study [10]. Watanabe et al. [18] concur with this conclusion where using multiple indicator dilution perfusion studies, in vitro rat hepatocyte uptake studies and in vitro rat liver microsome clearance measurements, they determine that the overall in vivo intrinsic clearance measurements approximate the in vitro uptake clearance measurements. They further predict in vivo human hepatic uptake clearance and suggest that these values approximate overall human intrinsic clearance measurements for atorvastatin and three other statins: pravastatin, pitavastatin and fluvastatin. These are excellent ground breaking analyses, however, I recently suggested [9] that further tests should be applied, incorporating efflux transporter and metabolism inhibition studies such as those carried out in our laboratory and recently reviewed [9], as well as studies where protein binding is changed. We suspect that other interactive processes may be at work, which have not yet been adequately defined in terms of Eq. 8. For example, the $\mathrm{CL}_{\mathrm{int} \text {,met }}$ portion of Eq. 8 is assumed to be independent of transport processes in the derivation of Kusuhara and Sugiyama [15] and in all other treatments of intrinsic metabolic clearance. As noted above, this is emphasized in the derivation of Eq. 3 as proposed by Rowland et al. [3], where the partition coefficient between unbound concentration in the measured systemic circulation and that in the eliminating organ is assumed to be constant. However, inhibition, induction or activation of transporters could potentially change this ratio so that in essence what we now call intrinsic metabolic clearance is not intrinsic, but rather varies with transporter changes. Our studies with atorvastatin in humans [10] not only showed inhibition by rifampin of atorvastatin uptake into the liver but also caused a change of the ratio of the atorvastatin lactone to the atorvastatin acid, thereby changing the amount of substrate available to the enzymes in the liver since, as we have shown earlier, metabolism by CYP3A4 of atorvastatin occurs via the lactone, not the acid [19]. Thus, I suspect that there are still chapters in the clearance book that need to be explicated and written. 


\section{Conclusion}

It is now 37 years since Professor Rowland introduced clearance concepts into pharmacokinetics. The impact has been, by 1972 standards, unbelievable and clearance has made pharmacokinetics an important underlying science in drug development, selection of dosage regimens in patients and in the regulatory process. As I have tried to point out here the development of clearance and its application is ongoing and will continue to serve an important role in our scientific understanding of drug disposition. It is only fitting that we recognize Professor Rowland's seminal and continuing contributions in this dedicatory issue.

Acknowledgments Supported in part by NIH Grants GM 75900 and GM 61390 and by an unrestricted grant from Amgen, Inc.

Open Access This article is distributed under the terms of the Creative Commons Attribution Noncommercial License which permits any noncommercial use, distribution, and reproduction in any medium, provided the original author(s) and source are credited.

\section{References}

1. von Bahr C, Alexanderson B, Azarnoff DL, Sjöqvist F, Orrenius S (1970) A comparative study of drug metabolism in the isolated perfused liver and in vivo in rats. Eur J Pharmacol 9:99-105

2. Krüger-Thiemer E, Diller W, Bünger P (1965) Pharmacokinetic models regarding protein binding of drugs. Antimicrob Agents Chemother 5:183-191

3. Rowland M, Benet LZ, Graham GG (1973) Clearance concepts in pharmacokinetics. J Pharmacokinet Biopharm 1:123-135

4. Wilkinson GR, Shand DG (1975) Commentary: a physiological approach to hepatic drug clearance. Clin Pharmacol Ther 18:377-390

5. Rowland M (1972) The influence of route of administration on drug availability. J Pharm Sci 16: 70-74

6. Benet LZ, Galeazzi RL (1979) Noncompartmental determination of the volume of distribution steady-state. J Pharm Sci 68:1071-1074

7. Klotz U, Avant GR, Hoyumpa A, Schenker S, Wilkinson GR (1975) The effects of age and liver disease on the disposition and elimination of diazepam in adult man. J Clin Invest 55:347-359

8. Sheiner LB, Rosenberg BG, Marathe VV (1988) Estimation of population characteristics of pharmacokinetic parameters from routine clinical data. J Pharmacokinet Biopharm 5:445-479

9. Benet LZ (2009) The drug transporter-metabolism alliance: uncovering and defining the interplay. Mol Pharmaceut 6:1631-1643

10. Lau YY, Huang Y, Frassetto L, Benet LZ (2007) Effect of OATP1B transporter inhibition on the pharmacokinetics of atorvastatin in healthy volunteers. Clin Pharmacol Ther 81:194-204

11. Zhang HX, Huang Y, Frassetto LZ, Benet LZ (2009) Elucidating rifampin's inducing and inhibiting effects on glyburide pharmacokinetics and blood glucose in healthy volunteers: unmasking the differential effects of enzyme induction and transporter inhibition for a drug and its primary metabolite. Clin Pharmacol Ther 85:78-85

12. Grover A, Benet LZ (2009) Effects of drug transporters on volume of distribution. AAPS J 11: 250-261

13. Sahin S, Benet LZ (2008) The operational multiple dosing half-life: a key to defining drug accumulation in patients and to designing extended release dosage forms. Pharm Res 25:2869-2877

14. Telbuvidine Package Insert (2009) www.accessdata.fda.gov/drugsatfda_docs/label/2009/022011s0 02lbl. Accessed 31 Dec 2009

15. Kusuhara H, Sugiyama Y (2009) In vitro-in vivo extrapolation of transporter-mediated clearance in the liver and kidney. Drug Metab Pharmacokinet 24:37-52 
16. Lau YY, Okochi H, Huang Y, Benet LZ (2006) Multiple transporters affect the disposition of atorvastatin and its two active hydroxy metabolites: application of in vitro and ex situ systems. J Pharmacol Exp Ther 316:762-771

17. Lau YY, Okochi H, Huang Y, Benet LZ (2006) Pharmacokinetics of atorvastatin and its hydroxy metabolites in rats and the effects of concomitant rifampicin single doses: relevance of first-pass effect from hepatic uptake transporters, and intestinal and hepatic metabolism. Drug Metab Dispos $34: 1175-1181$

18. Watanabe T, Kusuhara H, Maeda K, Kanamaru H, Saito Y, Hu Z, Sugiyama Y (2010) Investigation of the rate-determining process in the hepatic elimination of HMG-CoA reductase inhibitors in rats and humans. Drug Metab Dispos 38:215-222

19. Jacobsen W, Kuhn B, Soldner A, Kirchner G, Sewing K-F, Kollman PA, Benet LZ, Christians U (2000) Lactonization is the critical first step in the disposition of the 3-hydroxy-3-methylglutarylCoA reductase inhibitor atorvastatin. Drug Metab Dispos 28:1369-1378 